\title{
Wie dekolonial kann Kooperation sein?
}

\author{
Kritische Anmerkungen zu IKT-Interventionen im Globalen Süden
}

Simon Holdermann, Institut für Ethnologie, Universität zu Köln, Albertus-Magnus-Platz, 50923 Köln (s.holdermann@uni-koeln.de)

Konstantin Aal, Lehrstuhl für Wirtschaftsinformatik und Neue Medien, Universität Siegen (konstantin.aal@uni-siegen.de), (D) orcid.org/0000-0001-7693-7340

Projekte zu Informations- und Kommunikationstechnologien (IKT) in der Entwicklungszusammenarbeit im Globalen Süden operieren mit impliziten und expliziten Vorstellungen, Ansprüchen und Zielen. Die kritische Reflexion zu den Rahmenbedingungen, dem ethischen Status und den Konsequenzen solcher IKT-Interventionen und -Projekte kommt dabei oft zu kurz. Durch eine interdisziplinäre Perspektive und unter Rückgriff auf post- und dekoloniale Theorie können die Bedingungen und Partizipationsmöglichkeiten von „Nord-Süd-Kooperationen“ problematisiert und die ihnen zugrunde liegenden Begriffe, Konzepte und deren Konnotationen kritisch beleuchtet werden. Auf der Basis eigener Erfahrungen mit designorientierten Herangehensweisen in einem entwicklungs- und bildungspolitischen Projekt im Hohen Atlas in Marokko sollen diese Kritiken und Problemstellungen veranschaulicht und reflektiert werden. Indem eigene Vorannahmen, Erwartungen und Ansprüche auf den Prüfstand gestellt und Projektverlauf, Technikaneignung und interne Kommunikation nicht als gesetzt, sondern als prozesshaft und wechselseitig aushandelbar verstanden werden, können die Bedingungen für Kooperation in eine dekoloniale Richtung weisen.

\section{How decolonial can cooperation be?}

Critical remarks on ICT interventions in the Global South

Information and communications technology (ICT) interventions and development cooperation projects in the Global South operate with implicit and explicit ideas, expectations, and goals about the course of the project and cooperation. Critical reflection on the framework conditions, ethical status, and consequences of such ICT interventions and projects is often neglected. Through an interdisciplinary perspective and recourse to post- and decolonial theory, the conditions and participation possibilities of "North-South cooperation" can be problematized, and the underlying concepts and connotations can be critically examined. Based on our own experiences with design-oriented approaches in a development and education project in the High Atlas in Morocco, we will illustrate and discuss these critiques and problems. The conditions for cooperation can point in a decolonial direction by

This is an article distributed under the terms of the Creative Commons Attribution License CCBY 4.0 (https://creativecommons.org/licenses/by/4.0/)

https://doi.org/10.14512/tatup.28.2.s17

Submitted: 11. 02. 2019. Peer reviewed. Accepted: 13.05.2019 putting one's own assumptions, expectations, and demands to the test and by understanding project progress, technology appropriation, or internal communication not as given but as process-oriented and mutually negotiable.

Keywords: cooperation, Morocco, participatory design, ICT, postcolonial/decolonial critique

\section{Einleitung und Projektvorstellung}

Im Folgenden wollen wir anhand eigener Projekterfahrung über Problemstellungen bei Informations- und Kommunikationstechnologie-(IKT-)Interventionen im ,Globalen Süden ${ }^{11}$ reflektieren. Man befindet sich bei Projekten mit entwicklungspolitischem und gestalterischem Anspruch häufig im Spagat: Einerseits steht man handfesten Missständen gegenüber, die von Menschen vor Ort als solche empfunden und beschrieben werden (bspw. hinsichtlich Trinkwasser, Abfallbeseitigung, medizinischer Versorgung oder Bildung) und für deren Problemlösung sich lokale oder transnationale Akteur*innen aktiv einsetzen. Andererseits können Expertisen, normative Vorstellungen und Praktiken aus dem ,Globalen Norden ‘ Abhängigkeiten hervorbringen oder gar zementieren. Wie kann vor diesem Hintergrund das eigene Vorgehen, bzw. die gemeinsame Arbeitsweise, vorhandene Machtasymmetrien zu jeder Zeit kritisch zur Disposition stellen? Und auf welchen Wegen können Interventionsprojekte und Kooperationsbedingungen , dekolonialisiert' werden?

Um zu erklären, wie wir selbst mit den Fragen dieses Beitrags in Berührung gekommen sind und auf welchen eigenen Erfahrungen diese fußen, möchten wir zunächst unser eigenes Projekt beschreiben. Das interdisziplinäre Forschungsprojekt B04 im Rahmen des Sonderforschungsbereichs (SFB) 1187 „Medien

1 Bei der ersten Verwendung von ,Globalem Süden‘, bzw. ,Globalem Norden“ nutzen wir die Anführungszeichen, um darauf hinzuweisen, dass die Begrifflichkeiten nicht völlig neutral oder unumstritten sind (Comaroff und Comaroff 2012). 
der Kooperation" setzt sich zu einem Teil aus Sozio-Informatik (Wulf et al. 2018) und zum anderen aus Ethnologie zusammen. Gemeinsames Forschungsinteresse ist dabei, wie Medientechnologien und die sie umgebenden Medienpraktiken mit der weitreichenden Transformation einer Gebirgsregion im Hohen Atlas in Marokko zusammenhängen. Während die Sozio-Informatik auf Basis empirischer Forschung sozio-technische (Infra-)Strukturen in Form eines Computer Clubs (Aal et al. 2014) gestaltet und implementiert, verfolgt die ethnologische Forschung, welche Formen von Kooperation durch die Nutzung von Medientechnologie vor Ort entstehen und wie diese sich mit den lokalen politischen und sozio-kulturellen Begebenheiten und Ordnungen in Beziehung setzen lassen.

Die Intervention ist dabei zugleich eine Methode der sozio-informatischen Wissensproduktion und ein Projekt der Entwicklungszusammenarbeit. Als Teil eines Projekts in dem von der Deutschen Forschungsgemeinschaft (DFG) geförderten SFB, ist die DFG somit auch Geldgeber für die sozio-informatische Intervention. Der Umfang von Projekt und Intervention wird durch die im bewilligten Antrag abgesteckten Rahmenbedingungen vorgegeben. Darüber hinaus liegt der Ort der Intervention im wissenschaftlichen Erkenntnisinteresse und den eigenen Forschungsschwerpunkten begründet: Es handelt sich um eine stark verwandtschaftlich strukturierte Region, die von profunden sozio-ökonomischen, infrastrukturellen und technologischen Umbrüchen gekennzeichnet ist. In Verbindung mit detaillierten Ethnographien zu den lokalen Bezügen wird so eine Analyse der Transformationsprozesse und Herausbildung transnationaler Verknüpfungen in historischer Tiefe möglich.

\section{Rahmenbedingungen, Erwartungen und Herausforderungen der IKT-Intervention}

Insgesamt lassen sich drei Gruppen von Beteiligten identifizieren: die Forscher*innen aus Deutschland, die lokale marokkanische Nichtregierungsorganisation (NGO) und die Zielgruppe, d. h. all diejenigen, die durch das Projekt adressiert werden sollen. Alle Gruppen bringen indes ihre eigenen Erwartungen, Agenden und Zielvorstellungen mit sich.

Einige Rahmenbedingungen des Projekts waren bereits vor der eigentlichen Aushandlung mit den lokalen Kooperationspartnern über den konkreten Verlauf im Projektantrag der Forscher*innen fixiert. Auf Grundlage des Projektantrags konnten die Forscher*innen ihre Explorations- und Vorbereitungsreisen durchführen und vor Ort die lokale NGO als Kooperationspartnerin gewinnen. Im Zuge dessen wurden mit den Verantwortlichen der NGO der Verlauf und die Durchführung des gemeinsamen Projekts besprochen sowie eine vertragliche Kooperationsvereinbarung zwischen NGO und Universität unterzeichnet. ${ }^{2}$

2 Neben der Anschaffung von Technik wurden darin auch die Finanzmittel für den Betrieb des geplanten Computer Clubs (Stromkosten und Gehalt der Projektkoordinatoren der lokalen NGO) geregelt und festgehalten.
Im Projektantrag selbst wurden vorab Erwartungen der Forscher*innen formuliert: Der Computer Club könne eine Möglichkeit für lokale Ermächtigung (empowerment) sein. Für die lokale Bevölkerung solle damit eine Option geschaffen werden, an Debatten und Diskursen teilzunehmen, von denen sie bislang möglicherweise ausgeschlossen gewesen sei. Unterstützt durch die Projekt-Koordinatoren der lokalen NGO sollten die Teilnehmenden sich - alleine oder in Kleingruppen - in projektspezifischen Arbeiten Medientechnologien wie Tablets, Laptops und Kameras oder Navigationsgeräte und 3-D-Drucker aneignen (Rode et al. 2015). Um den Computer Club erfolgreich zu gestalten und zu etablieren, sollten insbesondere die methodischen Ansätze des Participatory Design (Bratteteig und Wagner 2012) und Grounded Design (Rohde et al. 2017) eingesetzt werden. Diese zielen im Kern darauf ab, die unterschiedlichen Beteiligten gleichwertig im Prozessverlauf - d. h. vom Design über die Implementierung bis hin zur Durchführung - einzubeziehen, sowie Relevanzen, Projektparameter und Zielvorstellungen gemeinsam vor Ort zu erarbeiten und aus der Empirie heraus zu entwickeln.

Die lokale NGO ist als Interessenvertretung für die Belange der Menschen gegründet worden und realisierte vor allem infrastrukturelle Gemeinschaftsprojekte, die auf Verbesserungen des alltäglichen Lebens abzielen, wie Befestigung von Bewässerungskanälen, Müllbeseitigung, oder Nachhilfeunterricht für Schulkinder. Aus diesem Grund hat sie eine hervorragende Reputation in der Bevölkerung und eine gute Vernetzung mit offiziellen Stellen. Der Computer Club wurde von der NGO in das bestehende Nachhilfe- und Förderprogramm für Schulkinder integriert.

Entgegen der Erwartungen der Forscher*innen an projektbezogene, kreative Arbeit mit und an der Technologie, wurde die Technik-Appropriation vor allem in einem Frontalunterricht-Setting angeleitet. Die Projektverantwortlichen wählten Schulkinder als Zielgruppe, da diese „,die Zukunft“ seien und noch bereit wären, sich neue Dinge erklären zu lassen. Gleichzeitig waren die Verantwortlichen auf Seiten der NGO davon überzeugt, dass es strikte Schulungsformate und klare Lernziele geben müsse, um den negativ bewerteten Konsequenzen der eigenverantwortlichen Projektarbeit (Ablenkung durch Smartphone und Facebook) vorzubeugen und einen professionalisierten Bildungsrahmen zu schaffen.

Kooperation $^{3}$ fand und findet im Rahmen des Projekts auf drei unterschiedlichen Ebenen statt: 1) zwischen den zwei Disziplinen Sozio-Informatik und Ethnologie im Forschungsprojekt selbst; 2) zwischen Forscher*innen und lokaler, marokkanischer NGO, mit der für die Intervention eng zusammen gearbeitet wird; 3) zwischen den Organisierenden des Computer Clubs (eigene Projektmitarbeiter*innen und lokale NGO) und der Zivilbevölkerung vor Ort. Hierbei treten durch die jewei-

3 Das spezifische Verständnis im SFB, das auch der Intervention zu Grund liegt, fasst Kooperation prozessual als die „wechselseitige Verfertigung gemeinsamer Abläufe, Ziele oder Mittel“ (Schüttpelz und Gießmann 2015, S. 39). 
ligen Kooperationsbedingungen unterschiedliche Reibungspunkte zutage: Die verschiedenen disziplinären Wissenschaftsverständnisse und -traditionen müssen in produktiven Dialog gebracht und Erwartungen, Agenden und Ziele sich gegenseitig deutlich gemacht sowie ausgehandelt werden. Diese gemeinsame Agenda bestimmt wiederum die konkreten sozialen und medientechnischen Praktiken im Computer Club mit den Teilnehmenden.

Kooperation, daran erinnern Star und Griesemer (1989), muss nicht auf einem gemeinsamen Konsens beruhen, sondern es kann durchaus unterschiedliche Interessen geben. In unserem Fallbeispiel war die Zusammenarbeit mit der NGO von Beginn an für die Kommunikation mit allen beteiligten Akteur*innen sowie für das Verständnis des lokalen Kontexts von größter Bedeutung. Gleichzeitig war die selbstverwaltete Appropriation des Computer Clubs im partizipativen Design des Projekts vorgesehen. Jedoch wich die Aneignungspraxis der kooperierenden NGO (Frontalunterricht, Fokus auf Schulkinder) von den im Projektantrag formulierten Erwartungen der Forscher*innen (Projektarbeit und maker-Gedanke, offen für alle) ab. Die eher politische Akzentuierung des Projektantrags erfuhr durch die Appropriation der Kooperationspartner*innen im Verlauf vielmehr eine entwicklungs- und bildungspolitische Stoßrichtung. Verschiedene Ansprüche an den Projektverlauf sowie Erwartungen hinsichtlich der Kooperationspartner standen sich widersprüchlich gegenüber.

Wenn die beantragten Projektvorstellungen von der tatsächlichen Umsetzung abweichen, ist dies durchaus ein Grund, einzu- gischer Artefakte, Infrastrukturen und Prozesse zentral miteinzubeziehen (Mainsah und Morrison 2014). Doch inwiefern steht dieser Ansatz im Widerspruch zur Planbarkeit und Finanzierung von Interventionsprojekten? Bevor wir diese Fragen in der Diskussion erneut aufgreifen, wollen wir uns zunächst dem postkolonialen Moment von ,Nord-Süd-Kooperationen“ widmen.

\section{Einwände post- und dekolonialer Kritik}

Bereits im Titel dieses TATuP-Themas finden sich die Begriffe „Entwicklung“ und „Fortschritt“, die auch in IKT-Interventionen und Projekten der Entwicklungszusammenarbeit verwendet werden. Diese Begriffe sind diskussionswürdig, weil sie eng mit dem Metanarrativ der Modernisierung verbunden sind. Sie sollen einerseits komplexe Zusammenhänge und Sachverhalte beschreiben, verleiten andererseits aber durch die mitgeführten Konnotationen dazu, den Blick auf die eigentliche Widersprüchlichkeit und Komplexität zu verstellen.

Vor allem Vertreter*innen der postkolonialen Theorie haben auf die Implikationen dieser Modernisierungsvorstellungen hingewiesen (Mignolo 2011; Bhambra 2014; Castro Varela und Dhawan 2015). Der zentrale Parameter von Modernisierung betrifft eine temporale (Ein-)Ordnung, die einen evolutionären Prozess beschreibt und entlang eines linearen Zeitverlaufs gedacht wird. Verbunden mit dem Eurozentrismus und den Vorstellungen über , die Anderen“ wird sie zur teleologischen Scha-

\section{Die Begriffe „Entwicklung“ und „Fortschritt“ sind diskussionswürdig, weil sie eng mit dem Metanarrativ der Modernisierung verbunden sind.}

greifen, um den Projekterfolg zu gewährleisten. Gleichzeitig widerspricht diese Steuerung dem partizipatorischen Ansatz, mit dem das beschriebene Interventionsprojekt verfahren möchte. In welchem Verhältnis stehen damit Kooperation und Partizipation?

Hier kommen Fragen nach den impliziten und expliziten Machtstrukturen von Kooperationsprojekten im Globalen Süden im Allgemeinen und von IKT-Interventionen im Speziellen auf. Denn wie und mit welchem Maßstab können und sollen konkurrierende Wissensformen bewertet werden? Wovon hängt der Projekterfolg ab und wer steuert letztlich das Projekt? Im Kern beschäftigt sich Participatory Design mit ebenjenen Fragen der Machtverhältnisse (Bannon et al. 2018), mit dem Ziel, die Kooperationspartner*innen als Expert*innen für eine angemessene Aneignung von IKT bzw. des Computer Clubs entsprechend der sozio-kulturellen Kontexte und lokalen Bedürfnisse wertzuschätzen und infolgedessen an der Entwicklung technolo- blone dafür, wie universeller Fortschritt erreicht werden könne: Entwicklung durch Modernisierung. Gemäß der impliziten Wertemaßstäbe dieser Vorstellung, wird , der Westen“ (Said 1981) dabei zugleich auf die ,oberste Entwicklungsstufe ' gesetzt, in Abgrenzung zu all jenen, die (noch) nicht ,modern ‘ - also ,entwickelt " - seien (Comaroff und Comaroff 1993; Ferguson 1999; Bhambra 2007). Dem Universalitätsanspruch klassischer Modernisierungsvorstellungen wurde durch die Beschreibung und Analyse von dynamischen, multiplen (Eisenstadt 2000; Boatcă und Spohn 2010) oder alternativen (Gaonkar 2001) Modernen eine Absage erteilt. Die Modernisierungslogik hält sich jedoch hartnäckig und schwingt auch in der Diskussion um die digitale Spaltung (digital divide) mit, wenn Zugang und Nutzung von IKT eine entscheidende Praxis zur Armutsbekämpfung dargestellt (Unwin 2017) oder gar als verheißungsvolle Motoren für nachhaltigen, ökonomischen Aufschwung und Erfolg verstanden werden (Graham 2018). 
Vorstellungen, in denen Zukunftsorientierung und Fortschrittshoffnung eingeschrieben sind, überführen die Dichotomien ,traditionell $\%$,modern ${ }^{\star}$ und , entwickelt $\%$, unterentwickelt ${ }^{\star}$ in eine aktualisierte Form: , digitalisiert $\%$,noch-nicht-digitalisiert'. Die Gefahr jener Vorstellungen relativer, Unterentwicklung' bestehen darin, dass durch sie Widersprüchlichkeiten rezenter Transformationsprozesse unzureichend simplifiziert und damit nicht vollumfänglich erfasst werden können (Bendix und Ziai 2015). Ein Beispiel dafür sind die Bedingungen der Mobilfunknutzung in der wirtschaftlich schwachen und vermeintlich ,rückständigen ' Bergregion in Marokko. Die Geschwindigkeit des mobilen Datenempfangs, die zwischen 3G und LTE

\section{Diskussion}

Im Laufe der IKT-Intervention im Rahmen des Forschungsprojekts ließen sich unterschiedliche Erwartungen und Widersprüchlichkeiten identifizieren, die auf Grenzen von Kooperation und Partizipation verweisen. Das Versprechen eines partizipatorischen Ansatzes scheint nie vollständig eingelöst werden zu können, weil die Antragstellung dem Projekt vorgelagert ist. Bevor die Kooperation als partizipatives Unterfangen startet, stehen Verlauf und Absicht bereits teilweise fest. Wie weit davon später abgewichen werden kann, bleibt eine projektspezifische Frage. Zudem ist fraglich, inwiefern ein partizipativer Ansatz überhaupt

\section{Es ist fraglich, inwiefern ein partizipativer Ansatz überhaupt gelingen kann, wenn Projekte aus antragsformalistischen Gründen im Globalen Norden designt wurden.}

schwankt, ist vor Ort im Hohen Atlas höher als in etlichen ländlichen Gebieten Deutschlands.

Des Weiteren sind Smartphones auch hier aus dem Alltag nicht wegzudenken und bereits für die Mehrzahl der marokkanischen Jugendlichen selbstverständlich. So nutzen unter ihnen $89 \%$ WhatsApp und sogar $95 \%$ Facebook. Dabei verwenden $92 \%$ der Jugendlichen, die einen Social Media Account besitzen, ein Smartphone um sich mit dem Internet zu verbinden (Gertel und Hexel 2018, S. 224-226).

Hierbei geht es nicht darum zu belegen, dass auch Menschen in Marokko ,modern' sind, sondern aufzuzeigen, dass diese Lebenswirklichkeiten durchaus als ,überraschend" oder exzeptionell aufgefasst werden - weil der angenommene ,Entwicklungszustand der Anderen ' dies nicht nahe legen würde. Im Gegensatz zu den tatsächlichen, ambivalenten und komplexen Lebenswirklichkeiten sind die imaginierten Umstände viel stärker von einer tiefsitzenden Perspektive der Defizite, Prekarität und sogar Ausweglosigkeit bestimmt (Ferguson 2006). Diese Annahmen wurden zum einen in kritischen Analysen postkolonialer Theoretiker*innen genealogisch auf den Kolonialismus zurückgeführt (Quijano 2007; Mignolo und Walsh 2018). Zum anderen seien, trotz der politischen Entkolonialisierung ehemaliger Kolonialgebiete, sowohl Vorannahmen und Konzepte als auch epistemische Praktiken größtenteils noch nicht dekolonialisiert (Mbembe 2016).

Wenn wir also anhand unseres eigenen Projekts fragen, wie dekolonial Kooperation sein kann, möchten wir damit Konzepte, Praktiken und Erwartungen in der Entwicklungszusammenarbeit im Globalen Süden auf den Prüfstand stellen. Ansprüche über Projektverlauf, Technik-Appropriation oder interne Kommunikation können nicht als gesetzte Fixpunkte verstanden werden, sondern müssen flexibel im gegenseitigen respektvollen Umgang erarbeitet werden. gelingen kann, wenn Projekte aus antragsformalistischen Gründen im Globalen Norden designt wurden und zu welchem Grad die kritische ,Offenheit' eines gemeinsamen Projektverlaufs gegeben sein kann. Kann ein solches Projekt überhaupt scheitern oder ist es zum Erfolg ,verdammt', gerade weil für die eigene Forschungsbiografie, aber auch im Rahmen der Antragstellung als Rechtfertigung und Verwendungsnachweis der Fördergelder immer entlang des Erfolgs argumentiert werden muss (Li 2007; Rottenburg 2009)? Damit sind es womöglich auch diese Messgrößen und der Bewertungsdruck solcher Projekte, die ein Stück weit die systemischen Bedingungen erzeugen, durch die die Verwendung von simplifizierenden oder gar (neo-)kolonialen Konzepten und Begrifflichkeiten begünstigt werden.

Eine gewisse Machtasymmetrie ist automatisch dadurch gegeben, dass ein solches Projekt stattfindet (Ferguson 1994; Escobar 1995). Schließlich sind es die Vertreter*innen aus dem Globalen Norden, die als Geldgeber fungieren, die Medientechnologie stellen und die aufgrund ihres Wissens und Expertise eine zentrale Rolle einnehmen. Gleichzeitig haben die Kooperation und das Projekt positive Konsequenzen - nicht nur nach den Bewertungsmaßstäben der Projektevaluation, sondern insbesondere nach jenen der Menschen vor Ort. So kann die lokale NGO ihre Position als wichtige zivilgesellschaftliche Akteurin stärken und Verbesserungen (ihren eigenen Wertevorstellungen entsprechend) herbeiführen, indem sie auf zuvor nicht verfügbare Ressourcen zurückgreifen kann. Zusätzlich ist es über den gemeinsam geschlossenen Kooperations-Vertrag auch mit rechtlichem Rückhalt möglich, Ansprüche geltend zu machen.

Bei IKT-Interventionen zu kooperieren, heißt sicher auch immer bereit zu sein, eigene Vorstellungen und Erwartungen über die gemeinsame Kommunikation und den Projektverlauf neu auszuhandeln. Sowohl fachinterne Methoden- und Theoriediskussionen der Ethnologie als auch der Sozio-Informatik haben 
Impulse zum Umgang mit eben jenen Fragen geliefert. Mit der Betonung der Koproduktion von wissenschaftlichen Erkenntnissen und deren unausweichlich politischen Komponenten haben in den letzten Jahren die Bestrebungen zugenommen, die eigenen Methoden (Smith 1999) bzw. die diesen zu Grunde liegenden epistemischen Praktiken (Verran 2001) zu dekolonisieren (Mbembe 2017; Savransky 2017). Zudem scheint auch der praxistheoretische turn in den Sozialwissenschaften (Schäfer 2016) vielversprechende Hinweise darauf zu bieten, welche alternativen Möglichkeiten bestehen, um sich in Kooperationszusammenhängen zu begegnen (Lassiter 2005; Hilton 2018).

Wenn die Praktiken und Konzepte ,der Anderen ' der eigenen Theoriebildung vorgeordnet werden, heißt das, auch die mit hineingetragenen Zuschreibungen und Vorannahmen zu reduzieren. So kann ein Möglichkeitsraum dafür entstehen, den gemeinsamen Projektverlauf als von Beginn an offen und prozesshaft anzusehen und als solchen auszuhandeln und zu gestalten. Hier setzt von anderer Seite der sozio-informatische Ansatz des Participatory Design an. Dieser räumt jenem gemeinsamen Aushandlungsprozess mit den lokalen Akteur*innen zu jedem Zeitpunkt der Intervention eine zentrale Stelle ein, indem die gestalterischen Ansprüche und Mittel zur Umsetzung auf die empirischen Begebenheiten vor Ort grundgelegt werden.

\section{Fazit}

Informiert durch kritische Einwände der post- und dekolonialen Theorie haben wir in diesem Beitrag die Kooperationsbedingungen und Partizipationsmöglichkeiten von IKT-Interventionen im Globalen Süden entlang eigener Projekterfahrungen hinterfragt. Projektlogiken und Argumentationsstrukturen, die solchen Interventionen zu Grunde liegen, können oft implizit eurozentrische und reduktionistische Konnotationen mit sich führen. Wir argumentieren dafür, das eigene Projekt stets kritisch zu reflektieren und Raum zu schaffen, unterschiedliche Erwartungshaltungen zu identifizieren, eine transparente und vertrauensvolle Kommunikationsebene zu etablieren und den Verlauf wechselseitig auszuhandeln. Im Projektantrag selbst sollte der angestrebten Offenheit des Forschungsverlaufs und der Notwendigkeit eines kollaborativen und partizipativen Projekt-Designprozesses Rechnung getragen werden. Hierzu sollten verstärkt interdisziplinäre Perspektiven und Konzepte, etwa aus kultur- und sozialanthropologischer, philosophischer oder sozialwissenschaftlicher Forschung, nutzbar gemacht und für angewandte Projektarbeit berücksichtigt werden.

Unserer Auffassung nach gelingen wichtige erste Schritte auf dem Weg zu einer Dekolonialisierung von Kooperation im Rahmen von IKT-Interventionen im Globalen Süden dadurch, Widersprüchlichkeiten nebeneinander zuzulassen: durch die Vielfalt methodischer Zugänge, durch ein transparentes und respektvolles Vorgehen und durch den wertschätzenden Umgang mit und die Betonung von historisch-spezifischem, lokalem und situiertem Wissen.

\section{Literatur}

Aal, Konstantin; Yerousis, George; Schubert, Kai; Hornung, Dominik; Stickel, Oliver; Wulf, Volker (2014): Come_in@palestine. Adapting a german computer club concept to a palestinian refugee camp. In: Naomi Yamashita und Vanessa Evers (Hg.): CABS'14. Proceedings of the $5^{\text {th }}$ ACM International Conference on Collaboration Across Boundaries. August 20-22, 2014, Kyoto, Japan. New York, NY: Association for Computing Machinery, S. 111-120.

Bannon, Liam; Bardzell, Jeffrey; B $\emptyset \mathrm{dker}$, Susanne (2018): Reimagining participatory design. In: Interactions 26 (1), S. 26-32.

Bendix, Daniel; Ziai, Aram (2015): Emanzipation durch Entwicklungspolitik? Einige Überlegungen zu Fragen globaler Ungleichheit. In: Momentum Quarterly. Zeitschrift für Sozialen Fortschritt 4 (3), S. 161-173.

Bhambra, Gurminder (2007): Rethinking modernity. Postcolonialism and the sociological imagination. Basingstoke: Palgrave Macmillan.

Bhambra, Gurminder (2014): Postcolonial and decolonial dialogues. In: Postcolonial Studies 17 (2), S. 115-121.

Boatcă, Manuela; Spohn, Willfried (Hg.) (2010): Globale, multiple und postkoloniale Modernen. München: Hampp.

Bratteteig, Tone; Wagner, Ina (2012): Disentangling power and decision-making in participatory design. In: Kim Halskov (Hg.): Proceedings of the $12^{\text {th }}$ Participatory Design Conference Research Papers - Volume 1. New York, NY: ACM, S. 41-50.

Castro Varela, María do Mar; Dhawan, Nikita (2015): Postkoloniale Theorie. Eine kritische Einführung. Bielefeld: Transcript.

Comaroff, Jean; Comaroff, John (Hg.) (1993): Modernity and its malcontents. Ritual and power in postcolonial Africa. Chicago, London: University of Chicago Press.

Comaroff, Jean; Comaroff, John (2012): Theory from the South. Or, how EuroAmerica is evolving toward Africa. In: Anthropological Forum 22 (2), S. 113-131. Eisenstadt, Shmuel (2000): Multiple modernities. In: Daedalus 129 (1), S. 1-29.

Escobar, Arturo (1995): Encountering development. The making and unmaking of the Third World. Princeton: Princeton University Press.

Ferguson, James (1994): The anti-politics machine. Development, depoliticization, and bureaucratic power in Lesotho. Minneapolis: University of Minnesota Press.

Ferguson, James (1999): Expectations of modernity. Myths and meanings of urban life on the Zambian Copperbelt. Berkeley: University of California Press.

Ferguson, James (2006): Global shadows. Africa in the neoliberal world order. Durham: Duke University Press.

Gaonkar, Dilip (Hg.) (2001): Alternative modernities. Conference on Alternative Modernities. Durham: Duke University Press.

Gertel, Jörg; Hexel, Ralf (Hg.) (2018): Coping with uncertainty. Youth in the Middle East and North Africa. Friedrich-Ebert-Stiftung. London: Saqi.

Graham, Mark (Hg.) (2018): Digital economies at global margins. Cambridge: MIT Press.

Hilton, Amanda (2018): Collaboration in anthropology. The (field)work of grounded practice. In: Cambio 8 (15), S. 113-126.

Lassiter, Luke (2005): Collaborative ethnography and public anthropology. In: Current Anthropology 46, S. 83-106.

Li, Tania Murray (2007): The will to improve. Governmentality, development, and the practice of politics. Durham: Duke University Press.

Mainsah, Henry; Morrison, Andrew (2014): Participatory design through a cultural lens. Insights from postcolonial theory. In: Ole Sejer Iversen, Heike Winschiers-Theophilus, Vincenzo D’Andrea, Claus Bossen, Maurizo Teli und 
Keld B $\emptyset \mathrm{dker}$ (Hg.): PDC 2014 reflecting connectedness. Proceedings of the $13^{\text {th }}$ Participatory Design Conference. October 6-10, 2014, Windhoek, Namibia. New York: ACM, S. 83-86.

Mbembe, Achille (2016): Ausgang aus der langen Nacht. Versuch über ein entkolonisiertes Afrika. Berlin: Suhrkamp.

Mbembe, Achille (2017): Critique of black reason. Durham: Duke University Press. Mignolo, Walter D. (2011): The darker side of Western modernity. Global futures, decolonial options. Durham: Duke University Press.

Mignolo, Walter; Walsh, Catherine (2018): On decoloniality. Concepts, analytics, and praxis. Durham, London: Duke University Press.

Quijano, Aníbal (2007): Coloniality and modernity/rationality. In: Cultural Studies 21 (2-3), S. 168-178.

Rode, Jennifer et al. (2015): From computational thinking to computational making. In: Kenji Mase, Marc Langheinrich, Daniel Gatica-Perez, Hans Gellersen, Tanzeem Choudhury und Koji Yatani (Hg.): Proceedings of the 2015 ACM International Joint Conference on Pervasive and Ubiquitous Computing. New York, NY: ACM, S. 239-250.

Rohde, Markus; Brödner, Peter; Stevens, Gunnar; Betz, Matthias; Wulf, Volker (2017): Grounded Design. A praxeological IS research perspective. In: Journal of Information Technology 32 (2), S. 163-179.

Rottenburg, Richard (2009): Far-fetched facts. A parable of development aid. Cambridge: MIT Press.

Said, Edward (1981): Orientalismus. Frankfurt am Main: Ullstein.

Savransky, Martin (2017): A decolonial imagination. Sociology, anthropology and the politics of reality. In: Sociology 51 (1), S. 11-26.

Schäfer, Hilmar (Hg.) (2016): Praxistheorie. Bielefeld: Transcript.

Schüttpelz, Erhard; Gießmann, Sebastian (2015): Medien der Kooperation. Überlegungen zum Forschungsstand. In: Navigationen. Zeitschrift für Medienund Kulturwissenschaften 15 (1), S. 7-55.

Smith, Linda (1999): Decolonizing methodologies. Research and indigenous peoples. London: Zed Books.

Star, Susan; Griesemer, James (1989): Institutional ecology, translations and boundary objects. Amateurs and professionals in Berkeley's Museum of Vertebrate Zoology, 1907-39. In: Social Studies of Science 19 (3), S. 387-420.
Unwin, Tim (2017): Reclaiming information and communication technologies for development. Oxford: Oxford University Press.

Verran, Helen (2001): Science and an African Logic. Chicago: University of Chicago Press.

Wulf, Volker; Pipek, Volkmar; Randall, Dave; Rohde, Markus; Schmidt, Kjeld; Stevens, Gunnar (Hg.) (2018): Socio-informatics. A practice-based perspective on the design and use of IT artifacts. Oxford: Oxford University Press.

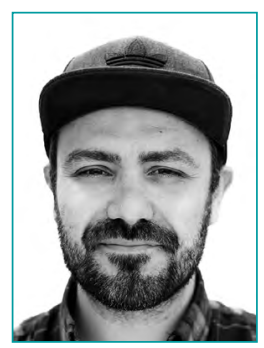

KONSTANTIN AAL

studierte Wirtschaftsinformatik an der Universität Siegen und ist seit Ende 2012 als Wissenschaftlicher Mitarbeiter am Lehrstuhl für Wirtschaftsinformatik und Neue Medien der Universität Siegen tätig. Seine derzeitigen Schwerpunkte liegen in der Forschung der Sturzprävention bei Senioren (iStoppFalls) sowie der Nutzung von sozialen Medien während des Arabischen Frühlings.

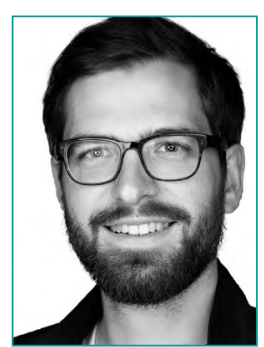

\section{SIMON HOLDERMANN}

ist seit 2016 Wissenschaftlicher Mitarbeiter am SFB 1187 „Medien der Kooperation“ der Universität Siegen und Doktorand im Fach Ethnologe/Kulturund Sozialanthropologe am Institut für Ethnologie und der a.r.t.e.s. Graduate School for the Humanities an der Universität zu Köln.

\section{politische ökologie}

Die Buchreien fuir Querdenker und Vordenterinnen

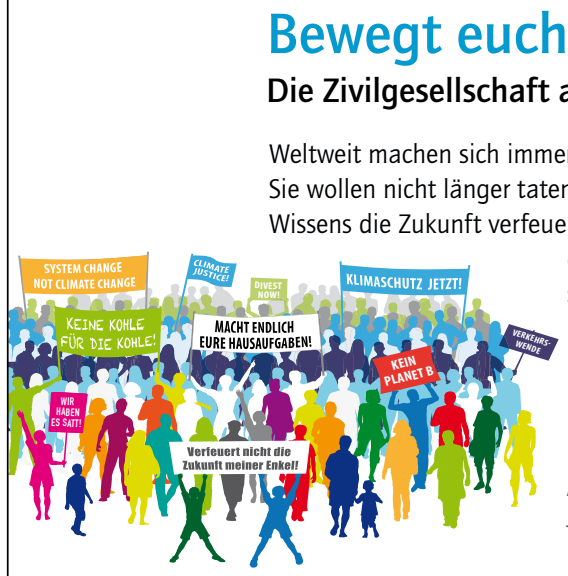

\section{Bewegt euch! \\ Die Zivilgesellschaft als Treiber der Dekarbonisierung}

Weltweit machen sich immer mehr Menschen für konsequenten Klimaschutz stark.

Sie wollen nicht länger tatenlos zusehen, wie die klimapolitisch Verantwortlichen wider besseren

Wissens die Zukunft verfeuern. So vielfältig die Aktionsformen auch sind, sie eint das Unbehagen gegenüber unserem fossil geprägten Wirtschaftsmodell, das mit seinem Wachstumsparadigma die planetaren Grenzen missachtet. Mehr denn je ist die Zivilgesellschaft als Mahnerin, Mittlerin und Motor für den Übergang in eine fossilfreie Zukunft gefragt.

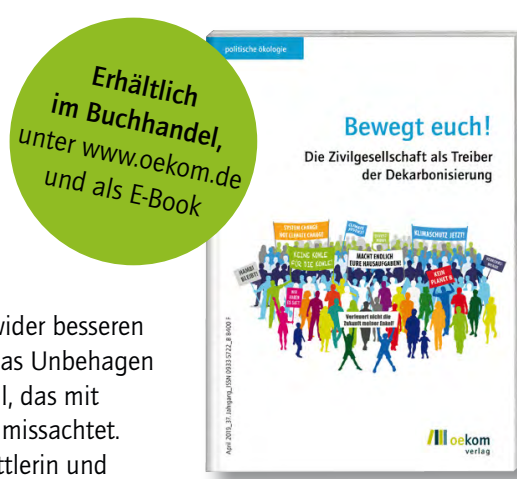

politische ökologie (Band 156): Bewegt euch! - Die Zivilgesellschaft als Treiber der Dekarbonisierung 144 S., 17,95 Euro, ISBN 978-3-96238-112-7 